\title{
Aménagements à buts multiples de grands fleuves
}

\author{
Pierre Savey, Directeur Général \\ Claude Cottereau, Directeur Adjoint de l'Ingénierie \\ Compagnie Nationale du Rhône
}

\section{Introduction}

Les aménagements des grands fleuves, dont le Rhône constitue la référence française, impliquent une conception à buts multiples pour tenir compte de l'importance et de la diversité de leurs potentialités.

Les services qu'ils peuvent rendre doivent être optimisés en fonction des objectifs poursuivis - principalement la production d'énergie, la navigation à grand gabarit, la protection contre les crues et les développements agricoles.

Cependant, l'intérêt relatif et les priorités de ces objectifs évoluent dans le temps tandis que de nouveaux objectifs apparaissent tels que les loisirs et la protection de l'environnement.

Les aménagements à buts multiples des grands fleuves doivent faire preuve de leur capacité à s'adapter à cette évolution, et tout particulièrement au respect de l'environnement naturel, voire à sa restauration.

\section{Conception adaptée aux objectifs des aménage- ments}

Trois objectifs essentiels dominent la conception des aménagements fluviaux à buts multiples :

- la production d'énergie ;

- la navigation à grand gabarit ;

- la protection contre les inondations et le développement de l'agriculture ou, plus globalement, l'aménagement du territoire ainsi protégé.

\subsection{La production d'énergie}

Si le seul objectif d'un aménagement fluvial est de produire de l'électricité et si la vallée est déserte ou presque, le type d'aménagement le plus radical et le moins coûteux est de construire un nombre minimal de barrages de grande hauteur équipés de puissantes centrales.

Cependant, il est courant de constater que les grands cours d'eau, quoique redoutés pour leurs sautes d'humeur et leurs crues dévastatrices, ont constitué depuis l'origine des temps des voies privilégiées de communication et ont donné lieu à des implantations humaines durables.

Dans l'exemple du Rhône, la construction de 2 ou 3 de ces barrages aurait entraîné la submersion de presque toute la vallée, des plaines les plus fertiles et de plusieurs villes importantes bâties sur les rives du fleuve (fig. l, page suiv.).

En outre, la conciliation avec les divers objectifs fixés à l'aménagement, par exemple ceux fixés à la CNR par la loi du Rhône de 1921, aurait été difficile, voire impossible : - le développement d'une navigation à grand gabarit implique de franchir de grandes dénivelées, souvent hors de portée des écluses classiques ; cette contrainte technique est génératrice de coûts de construction et d'exploitation considérables ;

- les plaines fertiles et les espaces qu'il est souhaitable de protéger et de mettre en valeur sont noyés sauf à réaliser des ouvrages d'endiguement importants, coûteux et consommateurs d'espace.

\section{Multi-purpose developments on large rivers}

The development of the large rivers, including the Rhone, is the French reference, implying multi-purpose objectives to take into account the importance and size of the potentialities of these rivers. Services that can by rendered must be optimized in accordance with objectives - mainly energy production, deep river navigation, protection against highwaters and agricultural developments. Nonetheless, the relative interest and priorities of these objectives change over time whereas new objectives appear such as leisure and environmental protection. Multi-purpose developments of large rivers must have the capacity to be adaptable to the above changes and especially to respect the natural environment, or even its restoration. 

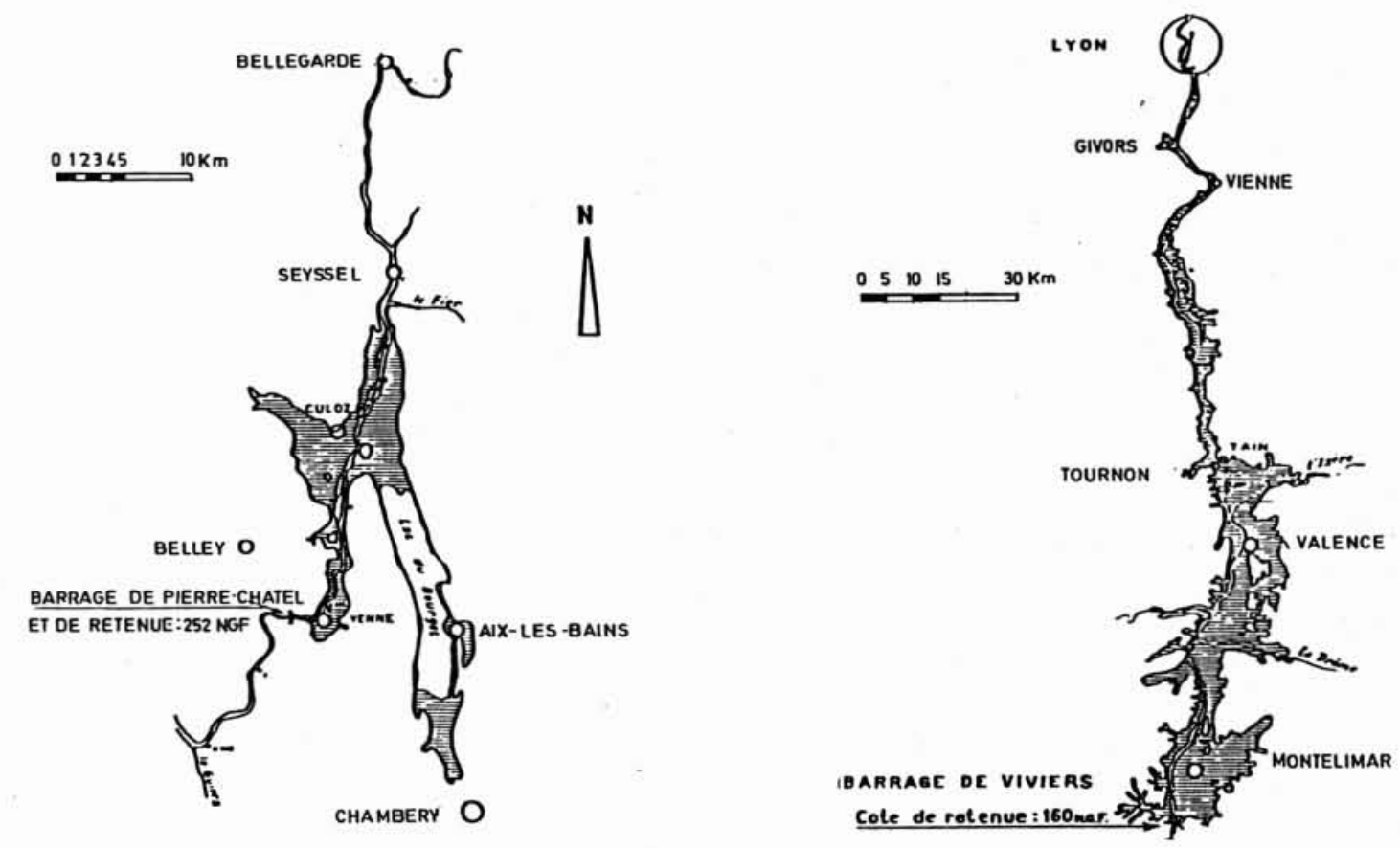

1.

1. Représentation schématique de grands barrages et de leurs retenues sur le Rhône.

2. L'aménagement à buts multiples du Rhône.

Enfin, sur le plan de l'environnement, les impacts sur les zones ripariales du fleuve, sur les espaces humides secondaires qui l'entourent, sur la qualité des eaux et leur autoépuration (effet de lac) et sur la faune aquatique, benthique ou piscicole, notamment les espèces migratrices, peuvent s'avérer considérables à long terme et tout à fait difficiles à réduire ou à compenser.

C'est pour toutes ces raisons qu'il y a intérêt à concevoir l'aménagement à buts multiples d'un fleuve comme une succession de paliers de basse chute dont les ouvrages sont réalisés soit dans le lit même du fleuve soit sur des dérivations de celui-ci.

Dans le cas du Rhône, la géographie de la vallée qui présente une succession de bassins alluviaux et de défilés rocheux, ainsi que la présence d'importantes implantations urbaines, ont été les facteurs déterminants du découpage des paliers, de leur niveau de retenue et de l'implantation des ouvrages principaux (fig. 2 et 3 ).

Au total, grâce à une combinaison judicieuse des retenues, des dérivations et des dragages de recoupement des chutes, il est possible de récupérer la majeure partie de l'énergie sauvage disponible pour produire de l'énergie électrique dans des conditions économiques optimales et en ménageant l'environnement naturel et humain de la vallée.

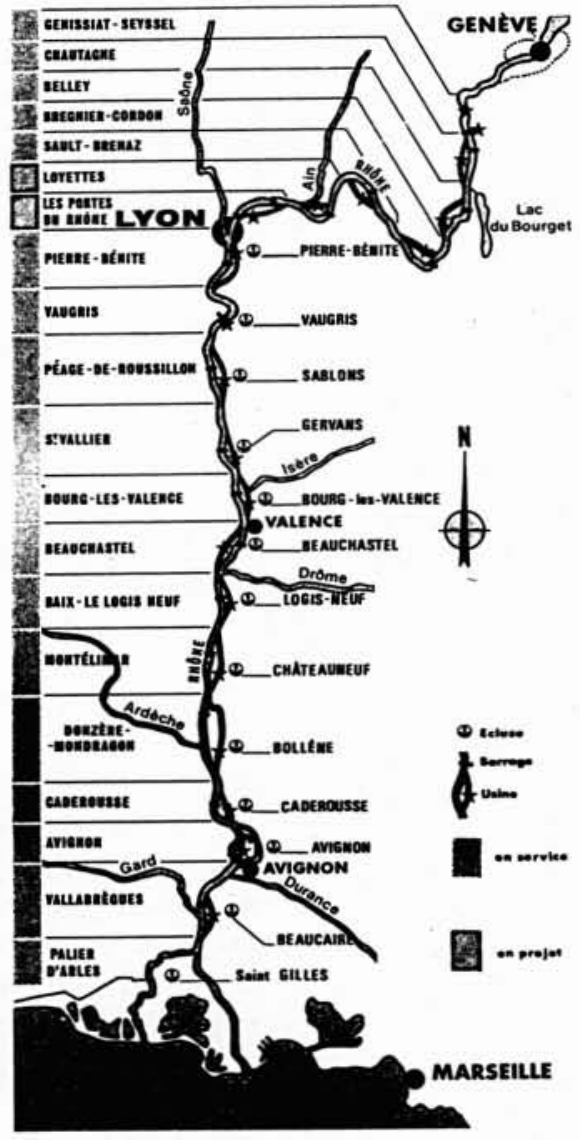

2. 


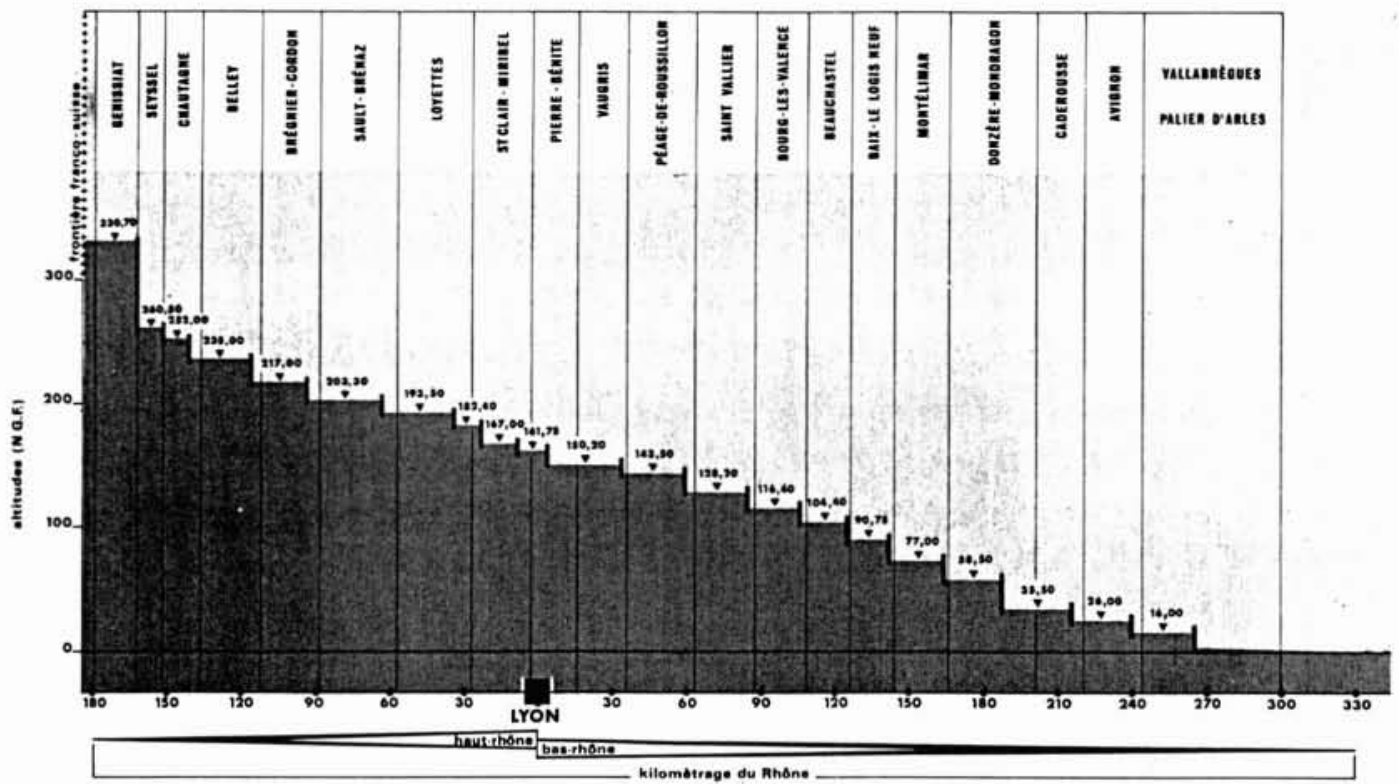

3. Profil en long de l'amènagement du Rhöne.

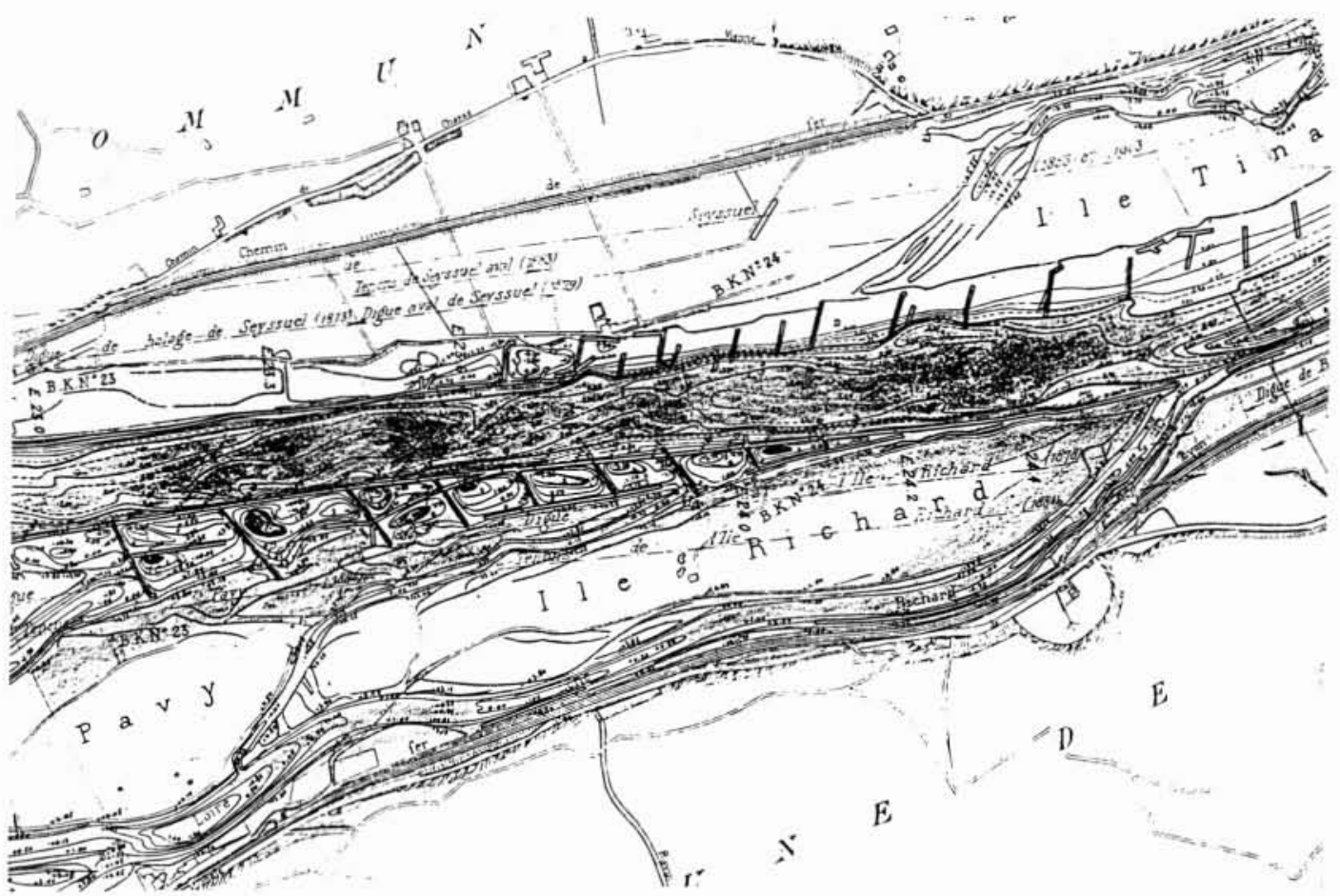

4. Aménagement à courant libre du Rhöne entre Lyon et Vienne.

\subsection{La navigation à grand gabarit}

Comme on le disait plus haut, les fleuves et les rivières ont constitué depuis toujours des axes de communication privilégiés que les hommes ont sans cesse cherché à améliorer.

Tout le long du Rhône, les travaux réalisés par la CNR ont mis à jour des vestiges d'implantations portuaires témoignant d'un commerce actif entre les divers établissements urbains de la vallée et entre ceux-ci et les villes de l'ensemble de la Méditerranée.

Les premiers travaux importants réalisés pour développer sur le Rhône une navigation moderne sont ceux de l'ingénieur de la navigation GIRARDON, effectués au $\mathrm{XIX}^{\mathrm{e}}$ siècle, travaux qui forcent l'admiration par la qualité de leur adaptation aux problèmes à résoudre et par leur continuité, si l'on songe aux moyens disponibles à l'époque.

Ce type d'aménagement dit "à courant libre » continue d'être utilisé dans de nombreux pays du monde entier.

Il consiste, généralement par des ouvrages en enrochements extraits de carrières voisines, plus rarement par des constructions en béton (panneaux de fond), à concentrer le débit de basses eaux dans le lit principal du fleuve (fig. 4). 


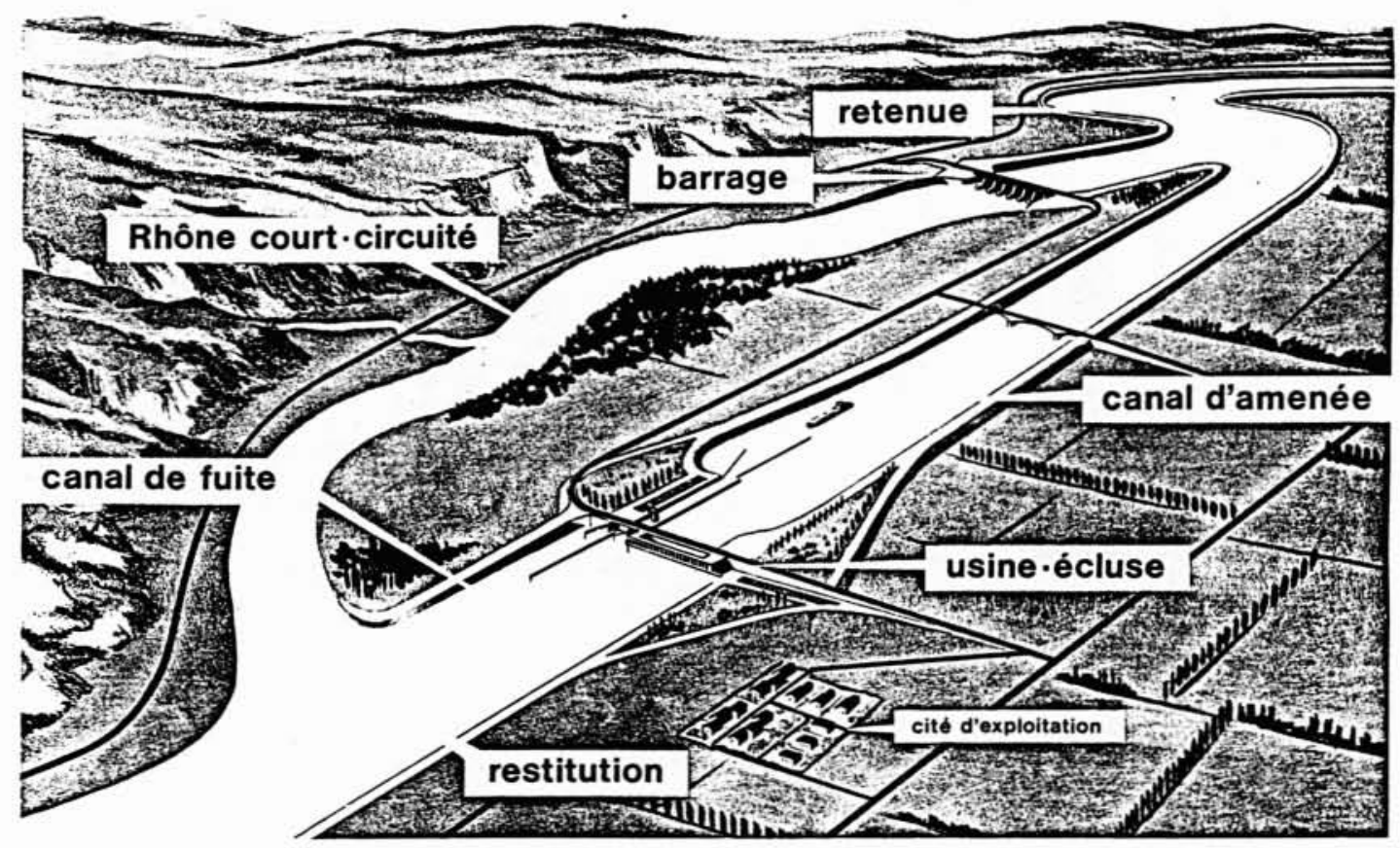

5. Schéma de l'aménagement type du Rhône.

L'objectif recherché est d'utiliser la force tractrice (ou érosive) des eaux pour auto-curer un chenal dans la partie extérieure des méandres et limiter la constitution de hautsfonds entre ces méandres.

Les structures mises en place doivent remplir leur rôle pendant les périodes d'étiage du fleuve sans constituer d'obstacles à l'écoulement des crues mais, au contraire et en complément, assurer pendant celles-ci une protection des berges.

\section{Pour cela :}

- les bras secondaires, souvent multiples dans le cas du Rhône, sont obturés par des "digues basses",

- les tronçons excessivement larges du lit principal sont rétrécis par des "épis plongeants", occasionnellement reliés entre eux dans les courbes prononcées.

Ce type d'aménagement est très peu agressif pour l'environnement naturel du fleuve. Il réduit les divagations du cours d'eau qui a tendance à abandonner ses bras secondaires qui se comblent peu à peu tandis que de nouveaux milieux se constituent à l'abri des courants violents en période de crues; les digues en enrochements deviennent autant d'abris à poissons.

Enfin, en réduisant ou supprimant les divagations du fleuve, l'aménagement à courant libre contribue à la sauvegarde des terres riveraines cultivables.

L'aménagement à courant libre a cependant, pour la navigation, des limites techniques étroites ; il est difficile de garantir, par ce système, des tirants d'eau d'étiage supé- rieurs à 1,60 ou 1,80 mètres ce qui est insuffisant pour une voie navigable à grand gabarit qui demande des tirants d'eau de l'ordre de 3 à 4 mètres.

Pour atteindre cet objectif, le seul moyen est la maîtrise des niveaux en période de faibles débits, maîtrise qui s'obtient par la mise en place de seuils ou de barrages mobiles de faible hauteur dans le lit du fleuve.

La navigation peut alors s'effectuer avec des tirants d'eau sensiblement constants quel que soit le débit du fleuve, au prix de l'installation d'une écluse au droit de chaque seuil ou barrage.

Il y a d'ailleurs souvent avantage, pour assurer la navigation lors des périodes de fortes eaux du cours d'eau, d'isoler celle-ci du lit principal en lui faisant emprunter un canal latéral.

Aucun débit, celui des bassinées mis à part, ne transite par le canal de navigation dont la conception est alors adaptée à l'usage qu'on en fait : protection anti-batillage des berges mais absence de protection contre les courants.

Au prix d'un accroissement de ses dimensions et d'un complément de protection, ce canal peut permettre d'alimenter une usine hydroélectrique, accolée à l'écluse de navigation, et ainsi concilier deux objectifs essentiels de l'aménagement du fleuve.

La maîtrise des niveaux du fleuve, quel que soit son débit, tant dans le lit principal que dans le canal latéral, permet d'assurer l'alimentation de prises d'eau pour les besoins agricoles, et le troisième objectif principal de l'aménagement se trouve ainsi atteint, au moins partiellement. 


\subsection{Protection contre les inondations et développement de I'agriculture}

On rencontre dans les plaines cultivées des grands fleuves, de nombreuses installations anciennes, parfois encore utilisées, ayant comme objectif la protection contre les inondations les plus fréquentes.

Il s'agit le plus souvent d'endiguements de faible hauteur incomplets, ouverts sur l'aval, complétés par un réseau complexe de canaux de drainage et d'ouvrages vannés, dont les manœuvres d'ouverture et de fermeture suivent des consignes laborieusement mises au point mais qui n "échappent pas pour autant à la contestation, les intérêts de l'amont n'étant pas toujours ceux de l'aval...

Ce type de protection, fréquent dans la vallée du Rhône et de la Saône, ne peut contribuer que faiblement au développement de l'agriculture ; de nombreuses cultures au rapport financier intéressant, comme le maraîchage, les fruits, le maïs, le soja, demeurent interdites à l'agriculteur.

Enfin, l'agriculture des vallées fluviales se heurte souvent à ce phénomène naturel qui veut que l'hydrologie présente des variations saisonnières qui sont parfaitement à l'opposé des besoins de l'agriculture : hautes eaux, voire crues au moment de la germination de printemps, étiages sévères et nappes phréatiques basses en fin d'été.

La mise en valeur rationnelle des riches espaces agricoles que constitue la plaine alluviale nécessite donc d'une part, une excellente protection contre les crues de fréquence au moins décennale et d'autre part la garantie d'une alimentation en eau satisfaisante pendant la période sèche soit par une stabilisation de la nappe phréatique, soit par des possibilités d'irrigation.

Les aménagements hydroélectriques de basse chute ou la canalisation pour la navigation imposent la plupart du temps la création d'endiguements latéraux qui satisfont à la première condition précédente, la maîtrise des niveaux d'eaux acquise à l'occasion de ces aménagements satisfaisant à la seconde condition.

\subsection{L'aménagement-type à buts multiples - l'exemple du Rhône}

Si l'on excepte le cas particulier du barrage de haute chute de Génissiat, l'aménagement du Rhône est constitué par une succession de paliers de basse chute dont le schématype comporte (fig. 5):

- un barrage à vannes mobiles qui relève le niveau du Rhône et crée vers l'amont une retenue de quelques kilomètres de longueur ;

- un canal de dérivation se développant en général dans la plaine alluviale et sur lequel sont implantés : une usine hydroélectrique exploitant la chute créée par le barrage et la dérivation, une écluse de navigation accolée à l'usine, des prises d'eau nécessaires à l'irrigation.

Ces ouvrages sont complétés en fonction des nécessités imposées par le site par:

- des endiguements bordés de contre-canaux de drainage protégeant les plaines riveraines contre les effets de la retenue et contre les crues. Cette protection s'exerce le plus souvent jusqu'à la crue de fréquence millénale (crue de projet) sauf lorsque l'écrêtement des crues impose le maintien de secteurs inondables des fréquences décennale ou centennale ;

- des dragages de recoupement des chutes dont l'intérêt est à la fois énergétique et pour la navigation ;

- des zones industrielles et portuaires.

Au total, le Rhône aménagé se compose de :

- 9 paliers (dont 6 en service) sur le Haut-Rhône pour une dénivelée totale de 170 mètres;

- 12 paliers tous en service sur le Bas-Rhône pour une dénivelée totale de 160 mètres environ.

\section{Evolution des objectifs et des priorités}

Les impératifs de l'économie régionale, voire nationale, ainsi que les intérêts, souvent divergents, des divers acteurs de cette économie influent largement sur la prise en compte des objectifs de l'aménagement et sur les priorités de ces objectifs les uns par rapport aux autres.

Cette évolution est également, dans une certaine mesure, une conséquence des "modes" et de la puissance de certains lobbies professionnels ou financiers, à l'écart de l'intérêt national.

Le Rhône, par exemple, bien qu'utilisé depuis deux mille ans comme axe de pénétration et bordé d'implantations urbaines et agricoles importantes, était encore, au début du $\mathrm{xx}^{\mathrm{e}}$ siècle, un fleuve sauvage, aux richesses pratiquement inexploitées et aux crues dévastatrices.

Les préoccupations principales des riverains, dont les initiatives sont à l'origine de la "Loi du Rhône " en 1921, étaient donc de se protéger contre les inondations et l'instabilité des berges, de disposer d'eau en abondance et à la bonne saison pour l'agriculture, et d'améliorer les conditions de navigation sur le fleuve trop rapide et insuffisamment profond à l'étiage.

Les travaux énormes que ces objectifs nécessitaient furent rendus possibles grâce au troisième objectif : la production d'électricité d'origine hydraulique.

Ce schéma, l'hydroélectricité comme moyen, la navigation et l'agriculture comme buts, a largement dominé les conditions de réalisation de l'aménagement du Rhône; il a cependant connu plusieurs remises en cause.

\subsection{La production d'énergie}

Il est devenu un lieu commun de souligner que l'électricité d'origine hydraulique :

- est non polluante; elle n'émet ni fumées, ni vapeur d'eau, elle ne réchauffe pas les eaux et n'y rejette aucun produit artificiel ;

- est peu bruyante ;

— bénéficie d'un combustible renouvelable gratuitement et indéfiniment ;

- est produite par des équipements de longue durée de vie ;

- est d'origine nationale et donc à l'abri des aléas géopolitiques. 
C'est en définitive la meilleure manière d'utiliser l'énergie solaire.

C'est par contre un moyen de production d'électricité très capitalistique qui nécessite de lourds investissements en comparaison d'autres moyens de production comme le thermique classique, le gaz et, dans une moindre mesure, le nucléaire.

Cette particularité est encore accentuée, voire déformée à l'excès, par les modes de calcul économique fixés par l'Etat ; le taux d'actualisation des calculs de rentabilité est celui du Plan soit $8 \%$ actuellement, après avoir connu des valeurs de 9 et même 10 (à titre de comparaison des pays européens voisins utilisent des taux d'actualisation compris entre 4 et $6 \%$ ).

La longue durée de vie des aménagements hydroélectriques n'est ainsi pas prise en compte et le montant élevé des dépenses de premier établissement pénalise lourdement et anormalement le taux de rentabilité de l'aménagement.

Malgré ce handicap, le plan initial de financement de l'ensemble de l'aménagement du Rhône par la seule production d'électricité a généralement pu être respecté, la CNR ne recevant de subventions ni de l'Etat, ni des collectivités locales, sauf dans les deux périodes suivantes :

— de 1966 à 1973, la baisse du prix du fuel, hardiment extrapolée jusqu'en l'an 2000 par des économistes plein d'optimisme, a nécessité que l'Etat contribue au financement de la part des ouvrages non producteurs d'énergie (navigation, agriculture); le choc pétrolier de 1973 a mis à mal les théories économiques de l'époque et a même contribué de manière décisive à l'équipement du HautRhône. Il a évité de voir se réaliser des aménagements " sans usine ", c'est-à-dire uniquement conçus pour assurer la continuité de la voie navigable et la protection contre les inondations, ce qui aurait actuellement de lourdes conséquences pour la gestion de la chaîne d'usines sur le Rhône ; - depuis 1980, les progrès techniques de la production nucléaire et le moindre développement de la consommation d'énergie ont gelé les équipements de production hydraulique; le retour à l'équilibre production/consommation est attendu pour 1995 environ.

\subsection{La navigation à grand gabarit}

On remarque une différence considérable entre la part du trafic fluvial dans le transport des marchandises entre la France (5\%) et l'Allemagne ( $25 \%)$, par exemple.

La France ne se trouve pas dans une situation originale par rapport à ses voisins pour ce qui concerne la nature du trafic et son évolution.

Cette différence provient seulement du sous-équipement de notre pays en infrastructures de voies navigables à grand gabarit aptes à faire communiquer divers bassins industriels.

Pourtant, le transport fluvial ne manque pas d'avantages par rapport à ses concurrents dont le plus direct est la route, puisque dans tous les pays le transport ferroviaire de marchandises régresse et que les sociétés de chemin de fer sont généralement déficitaires et subventionnées par la puissance publique.
Le transport fluvial :

- est d'abord le plus performant sur les longues distances pour les transports de charges lourdes ou encombrantes, les produits en vrac, les conteneurs de marchandises générales.

Un seul convoi poussé de 4000 tonnes équivaut à 100 wagons de 40 tonnes et à 200 camions de 20 tonnes. Cela signifie qu'on peut transporter 100000 tonnes par jour avec un convoi poussé par heure alors qu'il faudrait plus de 3 camions de 20 tonnes par minute pour obtenir le même résultat !

Cela explique également que la voie d'eau paraît toujours sous-utilisée alors qu'avec des trafics moindres, la route ou l'autoroute est en permanence au bord de l'apoplexie et fait naître l'impression qu'il faudrait encore plus de routes et d'autoroutes.

Cela n'est d'ailleurs pas faux sur l'axe Rhône-Saône où le trafic de poids lourds augmente de $10 \%$ par an depuis plus de trois ans;

- le transport fluvial est de ce fait le plus économique à la tonne transportée $=$ de l'ordre de $0,20 \mathrm{~F}$ d'économie par tonne kilométrique, par rapport à l'ensemble rail-route pondéré sur une distance moyenne de $500 \mathrm{~km}$;

- il est, pour les mêmes raisons de base, le moins polluant pour l'atmosphère ce qui revêt une certaine importance à l'heure actuelle (troubles respiratoires chez les riverains, destruction des forêts par les pluies acides, effet de serre,...);

- il est beaucoup plus sûr pour la sécurité des biens et des personnes que le transport routier;

- il est moins bruyant que la route ou le rail ;

- il est aussi le moins gaspilleur d'espace ;

- et enfin, il ne détruit pas les paysages mais peu permettre leur mise en valeur.

Le transport fluvial est, au total, le mode de transport le plus "écologique " à l'heure actuelle.

Il est cependant fort décrié à la fois par des défenseurs de l'environnement peu soucieux de la cohérence de leur discours et par des économistes porte-parole des lobbies concurrents.

Il est aussi victime des mêmes distorsions du calcul économique que les investissements de production hydroélectrique ; un taux d'actualisation trop élevé diminue anormalement la rentabilité apparente de la voie navigable.

Cela d'autant plus que le rôle structurant de la voie d'eau pour l'aménagement du territoire, vérifié tant sur le Rhône que dans les pays européens, n'est pas, ou peu, pris en compte dans les calculs.

Fort heureusement, après de longues années de désintéressement à l'égard de ce mode de transport, les pouvoirs publics viennent de se donner les moyens financiers d'investir largement et de manière durable dans les voies navigables à grand gabarit de la France, grâce à une taxe originale sur les utilisations multiples de l'eau.

La mise en cuvre de ce prélèvement financier est la meilleure reconnaissance a posteriori qui pouvait être donnée à la multiplicité des avantages offerts par la voie navigable à grand gabarit quant à son rôle fédérateur pour le développement des espaces riverains. 


\subsection{La protection contre les inondations}

La protection contre les inondations a toujours été un souci primordial des riverains des cours d'eau et, en conséquence, de leurs représentants : élus, syndicats agricoles,...

De tous temps, les riverains ont cherché à se protéger contre les crues les plus fréquentes, n'acceptant d'abandonner leurs terres qu'à des inondations de plus en plus exceptionnelles.

Cet objectif a connu une certaine "inflation", à l'époque où la croissance agricole était une nécessité économique nationale; il a fait la richesse de nombreuses plaines alluviales dans le monde et en particulier celle de la vallée du Rhône.

Le développement des irrigations, la stabilisation des nappes phréatiques, les remembrements, la restructuration des installations de transformation et de commercialisation des produits agricoles, grâce à l'aide financière des conventions agricoles ont parachevé l'efficacité de la mise en valeur de la vallée.

Il faut bien admettre que cette mise en valeur s'est faite quelquefois au détriment des zones humides naturelles, voisines du fleuve, et qu'il a fallu toute la résistance des associations de défense de la nature pour soustraire au " dieu maïs " quelques lônes et quelques espaces naturels particulièrement intéressants.

Il semble que depuis plusieurs années, on en soit revenu à une plus juste mesure.

L'amélioration des rendements avec l'aide des engrais et des pesticides, avec l'apparition de nouvelles variétés plus productrices et plus résistantes, les compléments apportés par les primeurs en provenance des pays du sud de l'Europe grâce à la facilité des échanges, contribuent à une affluence de produits sur les marchés alors que la consommation atteint ses limites; cette abondance s'accompagne d'une baisse des prix à la production. On observe donc une restructuration des domaines agricoles qui utilisent les meilleures terres tandis que celles de moindre valeur sont abandonnées; c'est la déprise agricole.

Dans le même temps, on redécouvre l'intérêt des inondations, voire leurs vertus, non seulement pour le maintien de la qualité des terres mais également pour la recharge des nappes phréatiques et la qualité écologique des zones humides situées à l'écart du niveau du fleuve.

Les allemands ont montré l'exemple en réalisant dans les endiguements du Rhin des ouvrages de noyage permettant de recréer des inondations dans les plaines riveraines qui en avaient été mises à l'abri par l'aménagement du fleuve.

Parallèlement, l'apparition croissante de nitrates et de traces de pesticides dans les eaux potables dont les nappes alluviales constituent souvent la ressource principale (plus de 600000 habitants sont ainsi alimentés par le Val de Saône) ont conduit les autorités à prendre des dispositions protectrices évitant cependant de spolier les propriétaires.

C'est l'esprit de "l'article 19 " du règlement CEE $n^{\circ} 797 / 85$ du 2 mars 1985 qui permet d'assurer un revenu convenable, en compensant leurs pertes, aux agriculteurs qui acceptent d'adapter leurs façons culturales (nature des cultures, pratiques traditionnelles de production) aux exigences spécifiques des zones sensibles afin d'entretenir ou améliorer leur environnement. Le Val de Saône et ses vastes prairies devrait être éligible des dispositions de cet article car il a été reconnu comme faisant partie du patrimoine naturel européen qu'il convient de protéger.

\subsection{Les loisirs nautiques et le tourisme fluvial}

De telles préoccupations étaient totalement absentes de la conception des grands projets d'aménagements fluviaux à buts multiples consacrés uniquement à une mise en valeur industrielle et agricole de la vallée.

Peu à peu, le contrôle des niveaux de l'eau, le meilleur accès au fleuve et le développement d'une civilisation des loisirs génératrice de retombées économiques complémentaires ont fait apparaître ces nouveaux objectifs.

Il est fréquent que ceux-ci soient maintenant pris en considération dès l'élaboration des projets, à l'initiative des collectivités locales en concertation avec le maître d'ouvrage.

Cette prise en compte précoce est génératrice d'économies pour les aménagements de loisirs qui bénéficient des avantages offerts par la présence d'engins de chantier, la disponibilité de matériaux...

On a assisté ainsi le long du Rhône, à la création de plusieurs ports de plaisance de 200 à 300 places, de nombreuses haltes nautiques et d'aménagements plus complets intégrant port, plage, piscine, stade d'eaux vives, lieux de pêche, village de vacances, restaurant et commerces adaptés.

L'aménagement de la voie navigable du Bas-Rhône a permis le développement du tourisme individuel, local ou de transit, et du tourisme fluvial collectif. Sur le HautRhône, des équipements spécifiques (écluse de plaisance, portiques transbordeurs) assurent le franchissement des ouvrages par les bateaux de plaisance et un équipement complémentaire des usines par des écluses de petit gabarit est à l'étude.

\section{Adaptation à l'environnement des aménagements récents}

La protection de l'environnement naturel est une prise de conscience relativement récente qui s'est manifestée par le développement des associations de défense de la nature et leur opposition grandissante et quasi systématique à toute modification du milieu existant, baptisé souvent de manière abusive de « naturel ».

Sous cette pression, les pouvoirs publics ont édicté un certain nombre de textes réglementaires destinés à encadrer la conception et l'exploitation des aménagements, notamment fluviaux.

La plupart du temps, ces textes n'ont que peu complété les cahiers des charges imposés aux concessionnaires, lesquels comportaient déjà des dispositions au moins aussi contraignantes pour les récentes réalisations. 
3.1. L'évolution des débits réservés du Rhône (débits minimaux de salubrité maintenus dans les tronçons de fleuve court-circuités par les dérivations) est riche d'enseignements à cet égard (fig. 6).

Sur le Bas-Rhône, les débits réservés apparaissent relativement faibles par rapport au module du fleuve: de l'ordre de la dizaine de $\mathrm{m}^{3} / \mathrm{s}$ en moyenne pour des modules compris entre 1000 et $1700 \mathrm{~m}^{3} / \mathrm{s}$.

En revanche, et bien avant l'application de la «loi pêche" de juin 1984 (qui d'ailleurs ne s'applique pas au Rhône...) des débits réservés bien supérieurs à ceux de cette loi ont été imposés sur le Haut-Rhône : entre 30 et $100 \mathrm{~m}^{3} / \mathrm{s}$ moyens annuels pour des modules de l'ordre de 350 à $450 \mathrm{~m}^{3} / \mathrm{s}$.

L'intérêt écologique de plusieurs secteurs du Rhône, à l'abri de l'urbanisation et des voies de communication (à la différence du Bas-Rhône) a été ainsi pris en compte, parfois d'une manière excessive si l'on en juge par les bilans des suivis hydrobiologiques après mise en service des aménagements (autre disposition des cahiers des charges). Les différences entre l'évolution de la faune benthique et piscicole dans des tronçons de Rhône court-circuité véhiculant $15 \mathrm{~m}^{3} / \mathrm{s}$ ou $100 \mathrm{~m}^{3} / \mathrm{s}$ restent tout à fait limitées = moindre effet de choc de la mise en service, moindre hétérogénéité des biotopes par rapport à la situation initiale du fleuve.

En tout état de cause, on ne note jamais de modification fondamentale des espèces piscicoles dominantes à l'état initial.

3.2. Le déplacement des poissons migrateurs du Rhône, dont l'espèce type est l'alose sur le Bas-Rhône, a posé dès l'origine des problèmes qui n'ont été - et ne sont toujours - que très imparfaitement résolus.

Plusieurs ouvrages (écluses ou échelles à poissons) ont été réalisés au droit des barrages et sur les seuils créés par les aménagements les plus avals. Malgré des transformations successives, la migration des aloses reste limitée ; des essais sont actuellement conduits à l'écluse de Vallabrègues pour tester diverses autres solutions.

En revanche, la migration des anguilles et des truites de mer paraît s'être maintenue.

En imposant des ouvrages de franchissement sur plusieurs seuils de l'aménagement du Haut-Rhône, les cahiers des charges ont permis à la CNR d'acquérir une certaine expérience dans la conception des échelles à bassins successifs, dans leur adaptation aux conditions particulières du site et dans le suivi de leur efficacité.

De nombreux piégeages effectués en période de migration ont prouvé l'utilité de ces ouvrages, même pour des individus de petite taille d'ordinaire moins robustes.

3.3. La préservation des milieux humides a été un souci récemment révélé dans le cadre des aménagements du Rhône.

Des dispositions particulières ont été adoptées dès la conception des chutes pour maintenir, voire améliorer, l'alimentation en eau de certaines lônes (ou bras morts) du Haut-Rhône : des études scientifiques ont montré l'effica-

\begin{tabular}{|c|c|c|c|c|c|c|c|c|c|c|c|c|c|}
\hline \multirow{3}{*}{ AMENAGEMENT } & \multicolumn{12}{|c|}{ DEBIT RESERVE $\left(\mathrm{m}^{3} / \mathrm{s}\right)$} & \multirow{3}{*}{ Moyen } \\
\hline & \multicolumn{12}{|c|}{ mensuel } & \\
\hline & $\mathrm{J}$ & $\mathrm{F}$ & $M$ & A & $M$ & $\mathrm{~J}$ & 5 & $A$ & $\mathbf{S}$ & 0 & $\mathbf{N}$ & 0 & \\
\hline CHAUTAGNE & $\leftarrow$ & & 10 & & 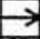 & $\leftarrow$ & & 20 & & & $\rightarrow$ & 10 & 15 \\
\hline BELLEY & $\kappa$ & & & & & & & & & & $\rightarrow$ & 25 & 35 \\
\hline BREGNIER - CORDON & $k$ & 80 & & & & $\epsilon$ & 150 & & & & & $\Rightarrow$ & 106 \\
\hline SAULT -BRENAZ & $\leftarrow$ & 20 & & & & 5 & 60 & & & & 20 & $\rightarrow$ & 30 \\
\hline LOYETTES & $\leftarrow$ & 60 & & & & $\rightarrow$ & $\leqslant$ & 80 & & $\leftarrow$ & 60 & $\rightarrow$ & 50 \\
\hline PIERRE-BENITE & $\leftarrow$ & 10 & & & & 20 & & & & & To & $\rightarrow$ & 16 \\
\hline $\begin{array}{c}\text { PEAGE DE } \\
\text { ROUSSILLON }\end{array}$ & $k$ & 10 & & & & 20 & & & & & 10 & $\rightarrow$ & 16 \\
\hline SAINT - VALLIER & $\leftarrow$ & 5 & & $\leftarrow$ & & 10 & & & & & 5 & $\rightarrow$ & 7 \\
\hline $\begin{array}{l}\text { BOURG-LES- } \\
\text { VALENCE }\end{array}$ & $k$ & 10 & & $\leftarrow$ & & $x$ & & & & & 10 & & $\mu$ \\
\hline BEAUCHASTEL & $\kappa$ & 10 & & $\leftarrow$ & & 20 & & & & & 10 & $\rightarrow$ & 14 \\
\hline $\begin{array}{l}\text { BAIX-LE- } \\
\text { LOGIS-NEUF }\end{array}$ & $k$ & 10 & & $\leftarrow$ & & 20 & & & & & 10 & & 16 \\
\hline MONTELIMAR & & & 10 & $\frac{560}{10}$ & & & & & & thon & & & 43 \\
\hline $\begin{array}{l}\text { DONZERE- } \\
\text { MONDRAGON }\end{array}$ & $k$ & & & & & & 60 & & & & & $\rightarrow$ & 60 \\
\hline CADEROUSSE & $\leftarrow$ & & & & & & 5 & & & & & $\rightarrow$ & 5 \\
\hline AVIGNON & 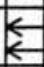 & & & & & $\frac{5}{100}$ & & SNC & & & & $\rightarrow$ & 400 \\
\hline VALLABREGUES & $\leftarrow$ & & & & & n & & & & & & $\rightarrow$ & 10 \\
\hline
\end{tabular}

6. Débits réservés des aménagements du Rhône.

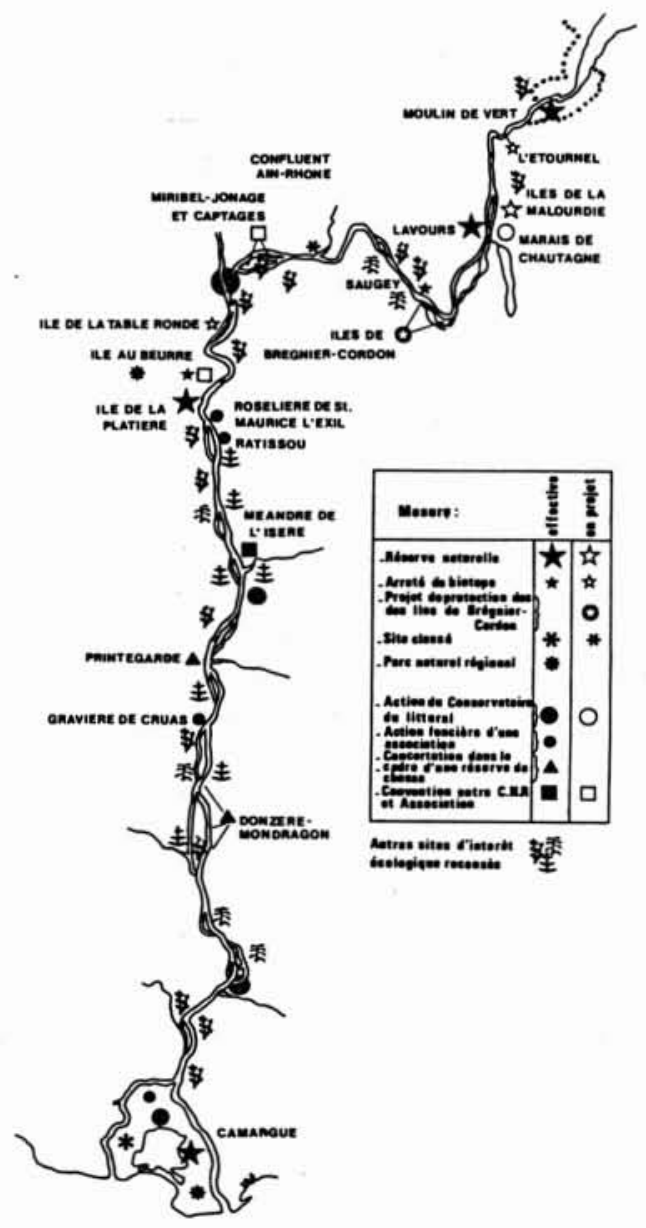

7. Les sites protégés du Rhône en 1988 


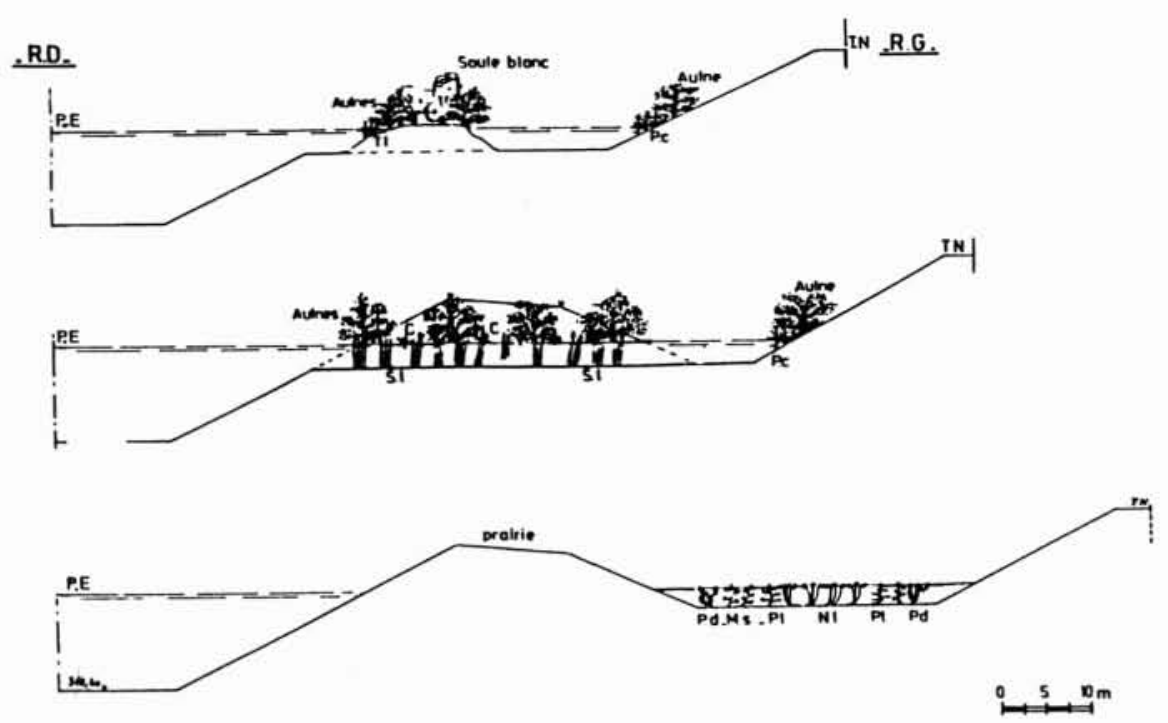

8. Profils en travers type des berges de la dérivation de l'Allan à Sochaux-Montbéliard.

cité de ces dispositions pour la régénération de ces milieux humides qui avaient tendance à se banaliser.

Des dispositions réglementaires ont été prises pour créer des réserves naturelles ou des secteurs protégés autour des milieux les plus intéressants : la Malourdie en Chautagne, les îles du Rhône à Brégnier-Cordon, la Platière et l'île du Beurre vers le Péage du Roussillon.

Un inventaire général effectué à l'occasion de cette prise de conscience a montré qu'en réalité les aménagements du Rhône, même les plus anciens, avaient sauvegardé tout le long de la vallée des zones humides dignes d'intérêt, oubliées à une époque oủ seule comptait la performance agricole, mais que l'on redécouvre aujourd'hui pour mieux les decrire et les mettre en valeur (fig. 7).

3.4. Le traitement des berges nouvellement créées par les aménagements a connu depuis une quinzaine d'années, sur le Rhône et sur d'autres ouvrages réalisés par la $\mathrm{CNR}$, des améliorations spectaculaires et pour tout dire uniques en leur genre.

Alors que sur les premières digues du Rhône on plantait abondamment le robinier (pseudo accacia) qui a l'avantage d'étouffer toute autre vie végétale, ou des herbacées communes, une approche écologique du problème de la revégétalisation des ouvrages en terre a été tentée.

Après une période d'essais approfondis en liaison avec des scientifiques du CNRS et de l'Université de Grenoble, des végétaux ligneux et herbacés ont été sélectionnés pour s'adapter aux diverses natures de sols, d'expositions, de pentes,... rencontrés sur les aménagements.

Les végétaux sont choisis parmi les espèces présentes initialement sur le site :

- arbres et arbustes de variétés naturelles, en formes libres, élevés spécialement dans une pépinière appartenant à la CNR:
- mélanges de graines d'herbacées sauvages récoltées dans des cultures particulières effectuées dans la vallée.

Commencée sur les dernières chutes du Bas-Rhône (Péage de Roussillon et Vaugris), cette technique avancée a été largement utilisée sur le Haut-Rhône ; elle est également employée pour réhabiliter progressivement certaines chutes anciennes du Bas-Rhône.

Enfin, elle a complètement dépassé la concession de la CNR pour s'étendre à la végétalisation d'autres barrages (Grand-Maison, St Egrève), de talus routiers et autoroutiers, de talus de voies ferrées dont le TGV, de sites miniers, de stations de ski,...

Ce mode de végétalisation étant limité aux parties hors d'eau des aménagements, il convenait de prolonger dans le milieu aquatique cette reconstitution du cadre naturel des cours d'eau.

Deux exemples illustrent cette renconquête :

* L'Allan, rivière affluente du Doubs, a fait l'objet, dans le cadre du projet de la liaison navigable Saône-Rhin à grand gabarit, d'une dérivation de quelques kilomètres.

Les berges de cette dérivation ont subi un modelage particulier mêlant sinuosités du tracé, risbermes à diverses profondeurs, zones abritées des courants,... afin de recréer des zones propices à assurer les fonctions essentielles de la vie piscicole : l'abri, la nourriture et la reproduction.

Pour compléter ces modelés du terrain, l'ensemble des berges a été planté de végétaux aquatiques récupérés dans des milieux humides voisins et mis en pépinière pendant la durée des travaux (fig. 8 ).

La partie hors d'eau des berges a reçu un traitement soigné par enherbement et plantations, selon les méthodes exposées plus haut, si bien que l'ensemble est particulièrement bien réussi et des pêches électriques de suivi ont montré l'efficacité de l'aménagement quant au développement de la faune piscicole. 


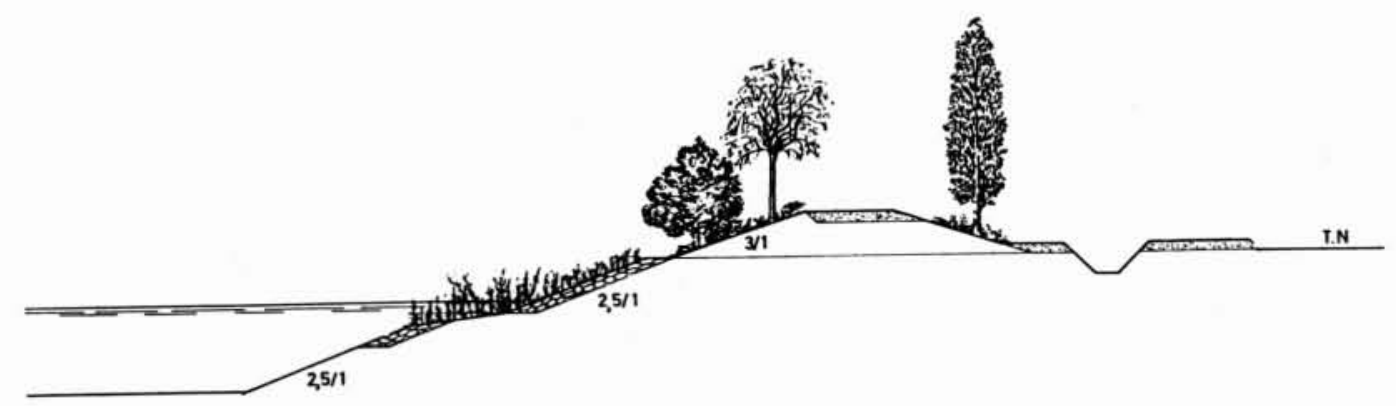

9. Profil en travers type de berge de la dérivation de la Saône à Mấcon.

* La dérivation de la Saône à Mâcon, autre ouvrage de la liaison navigable Rhin-Rhône, est actuellement en cours d'achèvement.

Les berges de ce canal de $4 \mathrm{~km}$ de longueur environ ont fait l'objet d'un traitement comparable à celles de la dérivation de l'Allan : risbermes noyées plantées de végétaux aquatiques de diverses variétés (fig. 9).

\section{Conclusion}

Imaginés et conçus avec des objectifs économiques qui étaient le reflet de leur époque, les grands aménagements fluviaux à buts multiples ont dû, pour pouvoir s'imposer et être menés à leur fin, s'adapter en permanence à l'évolution de ces objectifs et de leurs priorités respectives.

Cette nécessaire adaptation a été un puissant facteur de progrès technologique et économique dans de nombreux domaines.

Depuis une quinzaine d'années, la prise en compte de l'environnement dans la conception des projets et l'exploitation des ouvrages, d'abord négligée par les pouvoirs publics, a constitué une nouvelle exigence prioritaire.

Les aménagements à buts multiples ont su, dans ce domaine également, faire la preuve de leur faculté d'adaptation et de leur capacité à respecter le milieu naturel.

Ce nouveau défi imposé aux aménagements à buts multiples a eu deux résultats :
- il a fait notablement progresser la connaissance des grands systèmes fluviaux non seulement en France, mais dans le monde; ces progrès ont concerné aussi bien le milieu aquatique proprement dit (qualité des eaux, faune benthique, faune piscicole) que la dynamique des cours d'eau, leur évolution...;

- il a permis de montrer, à la faveur des suivis imposés à l'exploitation des ouvrages et grâce aussi aux études et inventaires effectués spontanément par les aménageurs, que les aménagements fluviaux à buts multiples tels que celui du Rhône, n'avaient pas eu de conséquences aussi catastrophiques qu'on voulait bien le dire, même après 20 ans de fonctionnement.

Il est bien certain que le visage général des vallées aménagées se trouve modifié et que de nouveaux milieux plus diversifiés se sont substitués aux anciens, plus homogènes.

Cependant, le fleuve continue de vivre et apporte en plus à ses riverains les richesses qu'on pouvait en attendre.

Il est bien sûr nécessaire de poursuivre à la fois les recherches scientifiques et le progrès des techniques pour améliorer l'intégration des aménagements fluviaux à buts multiples dans leur environnement.

Mais l'appréciation de l'impact de ces aménagements sur l'environnement doit aussi faire l'objet d'une réflexion globale à l'échelle de l'ensemble de la vallée concernée et même de la nation. Sans cet aménagement fluvial quels moyens devraient-ils être mis en œuvre pour produire de l'énergie, assurer les transports de marchandises et développer l'agriculture et quelles seraient leurs conséqences sur l'environnement naturel et humain? 